EDITORIAL

\title{
Kidney Injury in Sepsis: Fuel to the Fire
}

\author{
Binila Chacko
}

Indian Journal of Critical Care Medicine (2020): 10.5005/jp-journals-10071-23414

Two to three decades ago, acute kidney injury (AKI) requiring dialysis in a critically ill patient was considered to be associated with a poor prognosis. Have things changed since then? Is the Indian scenario different from the rest of the world?

Acute kidney injury affects close to $50 \%$ of critically ill patients world over ${ }^{1}$ and is associated with adverse outcomes. ${ }^{2}$ The incidence of AKI complicating stay in the intensive care unit (ICU) has risen over the past 20 years, possibly as a result of the increasing complexity of patients.

In this issue, Nandagopal et al. have explored the epidemiology of AKI in South India through a single-center cohort and have reported the impact of $\mathrm{AKI}$ on sepsis. ${ }^{3}$ Acute kidney injury was observed in $45.8 \%$ of this cohort, with sepsis being the inciting factor in over half these patients. These data appear consistent with international cohorts. ${ }^{2}$ The septic AKI subgroup had a higher requirement of dialysis albeit without a demonstrable difference in mortality when compared with nonseptic AKI. The authors reported a significantly higher mortality in the septic-AKI subgroup (25.8\%) when compared with the subgroup with sepsis alone (5.6\%). The mortality in both these subgroups was notably lower than other septic-AKI ICU cohorts ${ }^{4}$ and that reported in sepsis. ${ }^{5}$ The inclusion of lower disease severity as evidenced by the Acute Physiology and Chronic Health Evaluation (APACHE III) scores could explain this finding. It would have been preferable if data on the etiology of sepsis, etiology of AKI in the nonseptic group, and data on the type of patients (medical, surgical, and trauma), extent of organ dysfunction, and need for organ support (ventilator and hemodynamic) were available to aid in meaningful extrapolation and comparison of these data ${ }^{3}$ with other cohorts. This is important, given the heterogeneity of ICUs in our country.

The past three decades have witnessed much research and publications in the areas of sepsis and AKI. These syndromes share similarities in terms of the case definitions, disease burden, and mortality. Both sepsis and AKI have witnessed three changes in their case definitions. Sepsis has been defined per the Sepsis III criteria since 2016. Acute kidney injury likewise has gone through 3 definitions-the risk, injury, failure, loss and end-stage renal (RIFLE) and acute kidney injury network (AKIN) era are past and the kidney diseases: improving global outcomes (KDIGO) definitions are now used in clinical practice. ${ }^{1}$

With these changes, the burden of disease has been reported to be steadily rising; sepsis at an increase of $10 \%$ per year and AKI requiring dialysis has been found to have an annual increment of $2 \%$ from a US data set. ${ }^{5}$ This is probably related to the comprehensive definitions that have facilitated easy recognition and, second, due to changes in host factors over the years, i.e., older population with comorbidities, increasing use of contrast imaging, autoimmune diseases, cancers, and transplants.

Sepsis and AKI have also been reported to have a high mortality. Earlier on, families would be given a dismal prognosis whenever a critically ill patient needed dialysis in ICU. Even sepsis
Medical Intensive Care Unit, Division of Critical Care, Christian Medical College, Vellore, Tamil Nadu, India

Corresponding Author: Binila Chacko, Medical Intensive Care Unit, Division of Critical Care, Christian Medical College, Vellore, Tamil Nadu, India, Phone: +91 0416-2282693, e-mail: binilachacko@gmail.com

How to cite this article: Chacko B. Kidney Injury in Sepsis: Fuel to the Fire. Indian J Crit Care Med 2020;24(4):216-217.

Source of support:

Conflict of interest: None

and septic shock had mortality in excess of $80 \% 30$ years ago. ${ }^{6}$ A lot has changed since then. With better understanding of factors associated with poor outcomes, improvement in monitoring and organ support systems and adequate training of personnel, the mortality of sepsis is now closer to $20-30 \% .{ }^{4}$ The sepsisAKI combination continues to paint a morbid picture with 3-5 times higher mortality as demonstrated in the study by Kellum et al. ${ }^{3}$ and the current article. This increased mortality has been purported to be a result of electrolyte and acid-base disorders or impaired neutrophil function due to metabolic dysfunction. ${ }^{1}$ Outcomes are nevertheless better now as compared to earlier with reported mortality of $43.6 \%$ even in the septic AKI group requiring dialysis. ${ }^{5}$

As intensivists, how should we tackle this deadly combination? It is important to recognize, reduce, and respond appropriately.

It is crucial for intensivists to recognize risk factors for AKI in a critically ill patient, sepsis being the most common risk factor followed closely by hypovolemic shock and drug-related causes. ${ }^{1}$ These triggers should be appropriately and astutely managed. Since the incidence of AKI is high, it is all the more essential for intensivists to train both nurses and residents to recognize AKI early. This is necessary, given the higher odds of mortality with KDIGO stage 3 (odds ratio 6.884; 95\% confidence interval 3.876-12.228; $p<0.001$ ) as opposed to stage 1 or $2 \mathrm{AKI}^{5}$

The intensivist must additionally reduce and mitigate unwarranted triggers for AKI, e.g., nephrotoxic antibiotics, contrast, etc., and must aim at optimizing renal perfusion and systemic hemodynamics.

Responding and resuscitating appropriately are mandatory to prevent aggravation of the insult. While it is mandatory to treat the trigger, optimizing fluid therapy and organ perfusion are equally important. Maintaining an adequate volume status without overloading the interstitial compartment is vital. Liberal fluid strategy has been shown to be associated with a higher AKI incidence $^{7}(66 \%$ vs $58 \% p<0.001)$; the conservative approach, on the contrary, has been associated with lower incidence of AKI in the fluid and catheters treatment trial (FACTT $)^{9}(58$ vs $66 \%, p=0.007)$ and the conservative vs liberal fluid therapy in septic shock (CLASSIC) 
trial $^{10}$ (37 vs 54\%, $p=0.03$ ). A cautious approach avoiding overdiuresis is also recommended.

The optimal timing of renal replacement therapy (RRT) initiation in critically ill patients with AKI is another ongoing clinical dilemma. For critically ill patients with severe AKI but without the standard indications for RRT initiation, the appropriate triggers to initiate RRT are uncertain. The controversy between the two approaches to RRT, early anticipatory or a deferred approach, may be resolved with the results of the ongoing standard vs accelerated initiation of renal replacement therapy (STARRT)-AKI trial. ${ }^{11}$

Thus, in the setting of sepsis, early recognition of shock, adequate volume resuscitation, appropriate antibiotic therapy, avoidance of nephrotoxins, and maintenance of adequate renal perfusion can protect the vulnerable kidney from injury which in turn could influence outcomes of critically ill patients.

\section{References}

1. Ronco C, Bellomo R, Kellum JA. Seminar acute kidney injury. The Lancet 2019;394(10212):1949-1964. DOI: 10.1016/S01406736(19)32563-2.

2. Hoste EAJ, Bagshaw SM, Bellomo R, et al. Epidemiology of acute kidney injury in critically ill patients: the multinational AKI-EPI study. Intensive Care Med 2015;41(8):1411-1423. DOI: 10.1007/s00134-0153934-7.

3. Nandagopal N, Reddy PK, Ranganathan L, Ramakrishnan N, Annigeri $\mathrm{R}$, Venkataraman R. Comparison of epidemiology and outcomes of acute kidney injury in critically-ill patients with and without sepsis. Indian J Crit Care Med 2020;24(4):258-262.
4. Kellum JA, Chawla LS, Keener C, et al. The effects of alternative resuscitation strategies on acute kidney injury in patients with septic shock. Am J Respir Crit Care Med 2016;193(3):281-287. DOI: 10.1164/ rccm.201505-09950C.

5. Kalil AC, Johnson DW, Lisco SJ, Sun J. Early goal-directed therapy for sepsis. Crit Care Med 2017;45(4):607-614. DOI: 10.1097/ CCM.0000000000002235.

6. Sakhuja A, Kumar G, Gupta S, Mittal T, Taneja A, Nanchal RS. Acute kidney injury requiring dialysis in severe sepsis. Am J Respir Crit Care Med 2015;192(8):951-957. DOI: 10.1164/rccm.201502-0329OC.

7. Friedman G, Silva E, Vincent J-L. Has the mortality of septic shock changed with time? Crit Care Med 1998;26(12):2078-2086. DOI: 10.1097/00003246-199812000-00045.

8. Grissom CK, Hirshberg EL, Dickerson JB, et al. Fluid management with a simplified conservative protocol for the acute respiratory distress syndrome*. Crit Care Med 2015;43(2):288-295. DOI: 10.1097/ CCM.0000000000000715.

9. Liu KD, Thompson BT, Ancukiewicz M, et al. Acute kidney injury in patients with acute lung injury: impact of fluid accumulation on classification of acute kidney injury and associated outcomes. Crit Care Med 2011;39(12):2665-2671. DOI: 10.1097/CCM.0b013e318228234b.

10. Hjortrup $\mathrm{PB}$, Haase $\mathrm{N}$, Bundgaard $\mathrm{H}$, et al. Restricting volumes of resuscitation fluid in adults with septic shock after initial management: the CLASSIC randomised, parallel-group, multicentre feasibility trial. Intensive Care Med 2016;42(11):1695-1705. DOI: 10.1007/s00134-016-4500-7.

11. The STARRT-AKI Investigators. Standard versus accelerated initiation of renal replacement therapy in acute kidney injury: Study protocol for a multi-national, multi-center, randomized controlled trial. Can J Kidney Health Dis 2019;6(5):205435811985293. DOI: 10.1177/2054358119852937. 